\title{
POSITIVE EFFECTS OF PERIODIC SELF-REFLECTION USING "DAIFUKU-CHO" ON IMPROVEMENT OF STUDENTS'COMPETENCY
}

\author{
Yosuke Tohata, Akiko Takahashi, Hideyuki Kobayashi, Yoshiaki Rikitake \\ and Yoshikatsu Kubota \\ National Institute of Technology (KOSEN), Sendai College, Sendai, Miyagi, Japan. 4-16-1 Ayashi-Chuo, \\ Aoba-ku, Sendai-shi, Miyagi 989-3128, Japan
}

\begin{abstract}
KOSENs not only try to increase students' engineering abilities and literacy, which is the ability to use knowledge and skills, but also their competency, which we consider is the ability to apply knowledge and skills to any problem that might arise outside the walls of school. So the present study analyzes the relationship between a teacher's evaluation of students' self-assessment by "Daifuku-cho," which is used for teacher-and-student interaction in PE class, and their competency improvement using PROG test scores. The result confirmed that students' daily self-reflection and teachers' feedback on them are important, and the outcome of their daily self-reflection can quantitatively show the growth in their competency. In particular, we report that the interaction between students and their teacher through the use of "Daifuku-cho" enhanced their learning and also that by comparing what the students wrote in "Daifukucho," their grades and the results of PROG test, we suggest that the better students reflected themselves using "Daifuku-cho," the more their competency developed.
\end{abstract}

\section{KEYWORDS}

Self-assessment, Generic skills, Daifuku-cho, PROG test, PE Class

\section{INTRODUCTION}

School education around the world is rapidly transforming today. One-way lecture-based style used to be the teaching norm, in which teachers one-sidedly give students a lot of information and knowledge of academic subjects. Today active-learning style education is flourishing, in which students collaboratively find problems and try to solve them. To encourage students learn actively in the engineering field, we have carried out Active Learning (AL) [Niemi 2002, Hassan 2015], Mastery Learning (ML) [Kularbphettonga 2015, Shafie 2010, Kulik 1990], and 
Problem/Project Based Learning (PBL) [Rodri'guez 2015, Fernandes 2014] at Institute of Technology (KOSEN), Sendai College (Sendai KOSEN). Japanese KOSENs are schools where students from 15 to 20 years old receive five years of consistent engineering education. Sendai KOSEN is specialized in information and electronics, and offers various subjects in information and electronics field from basics to application such as basics in programming, artificial intelligence, IoT technology, and so on, to foster students' practical ability based on AL, ML and PBL.

In this educational system, we not only try to increase students' engineering abilities and literacy, which is the ability to use knowledge and skills, but also their competency, which we consider is the ability to apply knowledge and skills to any problem that might arise outside the walls of school. Especially, we believe that their experiences of problem-solving and group activity through AL and PBL will be highly effective to develop their competency, and have tried to evaluate their learning outcome in various measures [Takahashi 2016, Kubota 2017].

However, evaluation of students' academic achievements so far has been based on examination scores which just focus on their mastery of teaching contents and also their literacy and competency test scores. Teachers can learn how their students develop their abilities through them, but they are not sufficient for students themselves to work on continuous self-assessment, because they cannot assess what they are learning and how much they are growing in their abilities on a day-to-day basis at any time. Therefore, we need a framework to encourage students to assess their own daily growth and enhance their learning continuously.

The present study especially focuses on students' competency, and discusses how students' own review and reflection, or self-assessment, and teachers' feedback promote the students' personal growth, comparing individual student's growth using the annual test results of competency evaluation. The result confirmed that students' daily self-reflection and teachers' feedback on them are important, and the outcome of their daily self-reflection can quantitatively show the growth in their competency. In particular, we report that the interaction between students and their teacher through the use of "Daifuku-cho," a kind of shuttle card between teachers and students which contains records of students' learning and reflection and teacher's comments on them, enhanced their learning and also that by comparing what the students wrote in "Daifuku-cho," their grades and the results of PROG test, we suggest that the better students reflected themselves using "Daifuku-cho," the more their competency developed.

This paper consists of four sections. Section 1 introduced the background and purpose of this research. In Section 2 the related works and the proposal in this paper are explained. In Section 3 the comparison between the development of students' competency and their scores in "Daifuku-cho" is explained. Finally, Section 4 summarizes the current proposal and the future tasks to be tackled.

\section{RELATED WORKS AND PROPOSAL}

\subsection{Related Works}

In Japan, Cabinet Office is asking colleges and universities to develop human resources with high skills which full-fledged members of society are required to have. Responding to it, Ministry of Education, Culture, Sports, Science and Technology, Japan (MEXT) is promoting the introduction of AL into Japanese schools and colleges to cultivate human resources who can think and act on their own [MEXT 2014]. Many high schools and universities all over Japan are now working on AL. 
IADIS International Journal on Computer Science and Information Systems

As for the skills members of society are required to have, three basic skills and three wider skills are called "core skills" in the UK. The three basic skills, which comprise a national Key Skills Qualification, include that "communication," "numeracy or the application of numbers" and "use of information technology." The three wider key skills are "working with others," "improving own learning and performance" and "problem-solving" [Confederation of British Industry 1998]. In Canada, they are called "Employability Skills" [Conference Board of Canada], and they are classified into "Fundamental Skills," "Personal Management Skills" and "Teamwork Skills."

OECD also analyzed abilities of students and adults widely using various international surveys and existing studies. PISA Surveys investigate literacy, which means how people can make use of knowledge they acquired [OECD 2003], and DeSeCo is working on research about abilities and notions of foundation of sustainability as key competencies [OECD 2005]. In Hong Kong, the new Hong Kong three-year senior secondary curriculum (NSS) is aiming to improve students' abilities to think critically and creatively in the math classes. Ka-Cheng and et al. are trying to improve The Hong Kong Diploma of Secondary Education (HKDSE) Examination, the official evaluation approach, since they consider the exam inadequate to measure Generic skills [Leung 2014].

PROG test is used in many Japanese educational institutions for quantitative assessment of students' competency and it is possible to assess it by comparing with those which university graduates are required to have [Kawaijuku]. Ito et al. report that university students' Grade Point Average (GPA) and PROG have weak correlation [Ito 2014]. Fujiki et al. examine the effectiveness of teaching "Fundamental Competencies for Working Persons" advocated by Ministry of Economy, Trade and Industry, Japan, in KOSEN's first-year education using PROG test [Fujiki 2016]. As described above, attempts to improve generic skills in class and to measure their effectiveness have been carried out all around the world.

The use of shuttle-card "Daifuku-cho," which is used by students to review and reflect classes and to communicate with teachers, was proposed [Oda 1991]. "Daifuku-cho" has columns for students to fill in and ones for teachers to write some comments for students. After each class, students write what they felt in class or some questions in a column and submit it to the teacher. The teacher in return writes some comments and returns it to the students in the next class. "Daifuku-cho" enables teachers and students to interact, and students can review what they learned in the class and also learn from the teacher's comments. Kogo reports that the communication between students and teachers through "Daifuku-cho" leads to the students' introspective review of the class and higher class attendance rates [Kogo 2006]. Moreover, he reports the use of "Daifuku-cho" in e-learning courses where face-to-face communication is hard, and verifies that it contributes to reducing the distance between students and teachers. [Kogo 2007] "Daifuku-cho" proves effective to encourage students to look back on their daily learning. 


\subsection{Education in KOSEN}

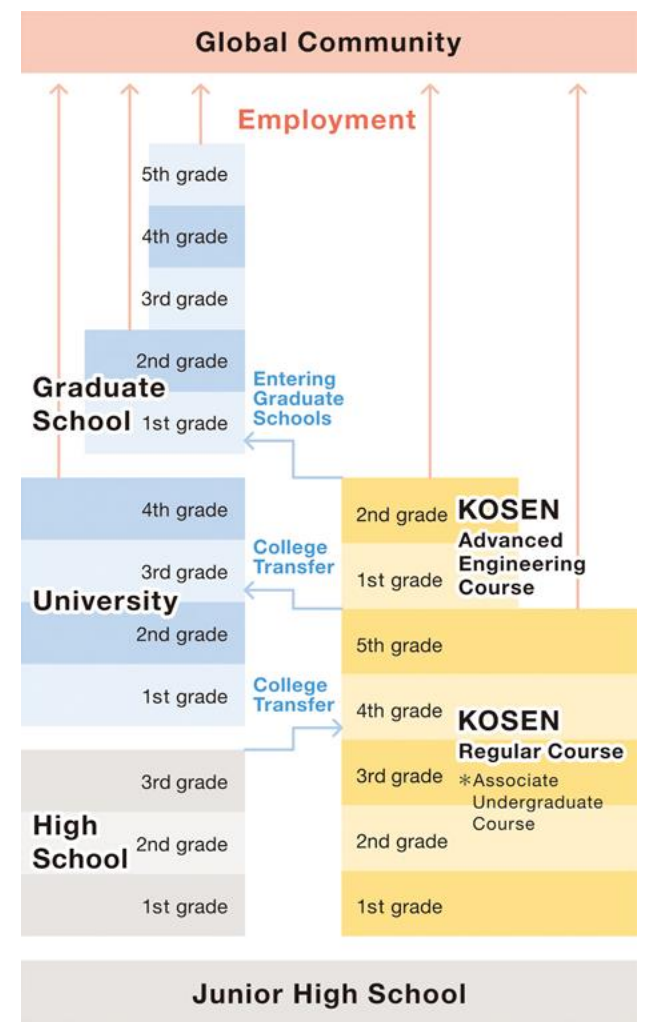

Figure 1. Process of going to higher education and finding employment in Japanese KOSENs

As described in Introduction (Figure 1), Japanese KOSENs are higher educational institutions where students from 15 to 20 years old receive five years of consistent education, mainly in engineering. Sendai KOSEN is specialized in information and electronics education, the curriculum for the students in this paper centers around software field consisting of subjects from basics in programming to advanced course of software development and their application. Our students also learn network technology and hardware technology including IoT, which are necessary to make use of software today. We try to encourage students not only to learn knowledge and techniques in their specialized field, but also to improve their "Fundamental Competencies for Working Persons" by teaching technology combining software, network and hardware in many AL, ML and PBL classes. 


\subsection{Proposal}

As described above, a lot of methods of evaluating competency and of improving learning outcomes by students' daily reflection have been proposed. The use of PROG is not adequate to assess students' growth on a day-to-day basis, because taking one test requires more than an hour, students need to take the test in regular intervals to assess their growth, or it takes a while to receive the result and feedback. The use of "Daifuku-cho" enables teachers to assess students" growth, but to evaluate the growth of their competency is difficult. Therefore, in the present study, by comparing the system to evaluate students' competency quantitatively and the effectiveness of students' day-to-day reflection, we propose a method to make students aware of the growth in their competency through classes. More specifically, we take physical education (PE) class as a test bed, in which we can see little difference among students' comprehension ability, and examine the relationship between the changes of students' competency in PROG and the grades based on students' reflection in "Daifuku-cho." We suggest that we can check part of students' growth in competency by using "Daifuku-cho."

\section{EVALUATION BY CORRELATION BETWEEN PROG TEST AND GRADES BASED ON "DAIFUKU-CHO"}

\subsection{Outline of Evaluation Using PROG Test and "Daifukucho"}

In the present study, we conducted a follow-up review of 2nd-year students (16-17 years old) and 4th-year students (18-19 years old) using PROG test and "Daifuku-cho." PROG test evaluates both "Literacy" and "Competency," and in this study we use "Competency" such as "ability to deal with environment surrounding in a practical manner" or "personal traits like policy of decision-making and action to cope with their surroundings" as one evaluation index, because it is more important than "Literacy" in "Fundamental Competencies for Working Persons." We used "Daifuku-cho" shown in Figure 2 as the other evaluation index and focused on PE class, in which each student's understanding does not lead to their grades straightforward. In the present study, we will show the effectiveness of "Daifuku-cho" as a tool for students to review their daily learning by examining the correlation of these two indices.

\subsection{Structure of "Daifukucho"}

"Daifuku-cho" is a double-sided, A4-size thick sheet of paper and 15 columns for one semester are printed on it. As shown in Figure 2, it consists of parts (a)-(f).

(a) Student ID: grade, class, student number, and name

(b) Class number and Date: the date of the class

(c) Goal of class: Students describe their personal goal of the class, what and how they are going to work on in the class, how they are going to improve themselves and contribute to the class, and so on.

(d) Review of class: The most important part of "Daifuku-cho." In terms of their own goal and class contents, students write: 
- How they understand the present state they are in and identify the problems: how far they have achieved or have not achieved.

- Setting up the tasks to achieve their goals: What factors are helping their achievement or preventing them, and what tasks are needed to solve the problems they are facing.

- Reflecting and describing their practices and challenges: To overcome the factors which preventing their achievement, what resources and methods they can try. Questions to the teacher or comments on the class.

(e) Teacher's feedback to what the student wrote. Adding special knowledge or answering the student's questions.

(f) Assessment: Teacher assesses what the student wrote in (d), whether they are helpful to take concrete steps to achieve their goals, whether they think logically and so on, on a scale of one to four and the full marks in one semester are 60.

In the present study, to have them think and reflect on their own, students are required to write by hand, not electronically, and teachers write comments by hand.

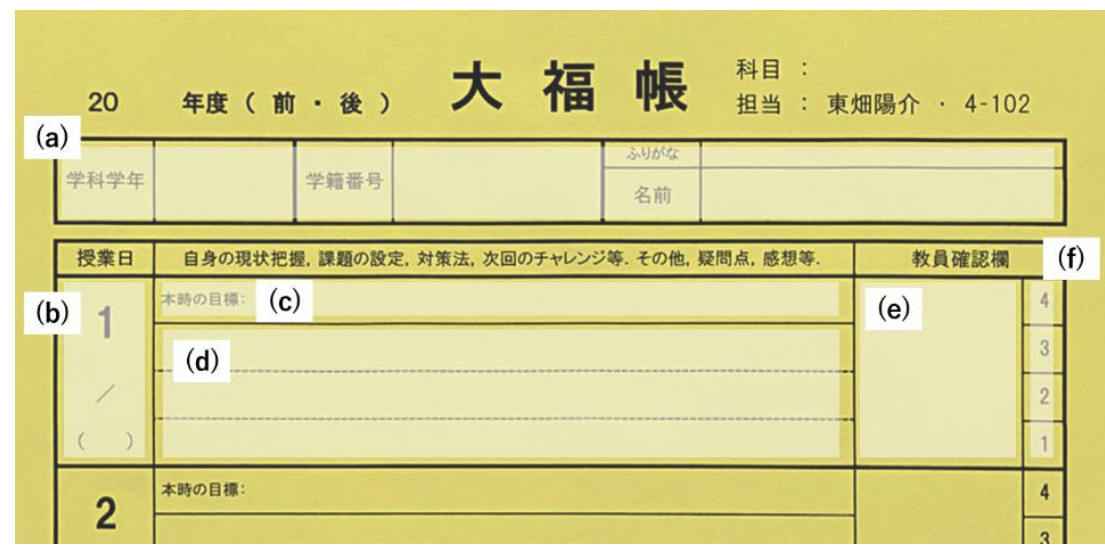

Figure 2. Structure of "Daifuku-cho" utilized in PE class

\subsection{Lesson Structure}

The subject of the present study is "Daifuku-cho," which is used in PE classes for 2nd-year students of KOSEN (16-17 years old) and 4th-year students (18-19 years old). Tables 1 show the contents of the lessons. In these lessons, a lof of group activities were done, to improve students' competency, in addition to usual contents of PE class aiming to improve students' athletic abilities and skills in each sport. For example, in golf or tennis lessons, students exercise together to improve their skills according to the plan which the teacher set in advance.

In group activities, students work on what sport they like to play or like to create in groups. They discuss, make plans, and implement them on their own. For example, 2nd-year students worked on creating a new sport by reference to existing sports (goal-style, net-style, baseballstyle, target-style, record-breaking-style, and so on). They have to consider target players' readiness to learn new rules, rules to add or delete, or minimum safety to ensure. They discuss from several points of view, decide rules of a new sport, play it on a trial basis in groups, and check how effective the rules are or how safe the sport is. Finally, they give a presentation of 
IADIS International Journal on Computer Science and Information Systems

the sport and by playing it in class together, they can get feedback from their classmates. 4th-year students manage their own PE classes based on the sports they have played, for example, they decide what sport they play in what group in a 90-minute lesson.

In these activities, the PE teacher do not give direct instructions but acts as a facilitator, just giving a few pieces of advice when asked or raising students' awareness in interaction. Basically, students have to think and act voluntarily involving other students, which goes deeper than using "Daifuku-cho" and is adequate to check students' competency.

Table 1. Outline of 2nd-years' and 4th-years' PE classes

\begin{tabular}{|c|c|}
\hline \multicolumn{2}{|c|}{ Topics } \\
\hline \multicolumn{2}{|c|}{ Creating a new sport } \\
\hline 1 & Grouping, Creating an original sport \\
\hline 2 & Simulating the original sport and improving it \\
\hline 3 & Group presentation and trial practice (1) \\
\hline 4 & Group presentation and trial practice (2) \\
\hline 5 & Group presentation and trial practice (3) \\
\hline 6 & Group presentation and trial practice (4) \\
\hline 7 & Summary, Sports in engineer's life \\
\hline \multicolumn{2}{|c|}{ ※ The author was in charge of 7 sessions of 1 semester course } \\
\hline \multicolumn{2}{|c|}{ 4th years' } \\
\hline \multicolumn{2}{|r|}{ Topics } \\
\hline \multicolumn{2}{|l|}{ Golf } \\
\hline 1 & Understanding the rules, Learning how to grip a club and swing it, Iron club \\
\hline 2 & Iron club, Wood club \\
\hline 3 & Target bird golf, Putting \\
\hline 4 & Playing rounds in campus (1) \\
\hline 5 & Playing rounds in campus (2) \\
\hline \multicolumn{2}{|c|}{ Tennis and rubber-ball tennis } \\
\hline 6 & Understanding the rules, Learning how to grip a racket and swing it \\
\hline 7 & Forehand stroke and backhand stroke \\
\hline 8 & Service, volley and smash \\
\hline 9 & Rally game (1) \\
\hline 10 & Rally game (2) \\
\hline \multicolumn{2}{|c|}{ Voluntary program } \\
\hline 11 & Elective sport (1) \\
\hline 12 & Elective sport (2) \\
\hline 13 & Elective sport (3) \\
\hline 14 & Elective sport (4) \\
\hline 15 & Elective sport (5) \\
\hline
\end{tabular}

※ In Voluntary program, each student or each group plan and implement a sport, including equipments and time schedule. 
POSITIVE EFFECTS OF PERIODIC SELF-REFLECTION USING "DAIFUKU-CHO” ON IMPROVEMENT OF STUDENTS'COMPETENCY

\subsection{Comparison between the Results of PROG Test and the Evaluation by "Daifuku-cho"}

Table 2. Each student's PROG competency and grade in Daifuku-cho

\begin{tabular}{|c|c|c|c|c|c|}
\hline \multirow{2}{*}{$\begin{array}{l}\text { Student } \\
\text { ID }\end{array}$} & \multicolumn{3}{|c|}{ Competency } & \multicolumn{2}{|c|}{ Daifuku-cho } \\
\hline & 2nd & $3 r d$ & 4th & 2nd & 4th \\
\hline 1 & 5 & 5 & 6 & 0.89 & 0.92 \\
\hline 2 & 5 & 5 & 6 & 0.89 & 0.93 \\
\hline 3 & 5 & 5 & 6 & 0.50 & 0.92 \\
\hline 4 & 2 & 5 & 4 & 0.93 & 0.85 \\
\hline 5 & 5 & 5 & 6 & 0.79 & 0.83 \\
\hline 6 & 4 & 4 & 5 & 0.89 & 0.97 \\
\hline 7 & 5 & 6 & 6 & 0.93 & 0.83 \\
\hline 8 & 2 & 2 & 3 & 0.96 & 0.88 \\
\hline 9 & 1 & 1 & 7 & 0.89 & 0.88 \\
\hline 10 & 4 & 5 & 6 & 0.93 & 1.00 \\
\hline 11 & 4 & 5 & 5 & 0.68 & 0.77 \\
\hline 12 & 3 & 2 & 6 & 0.75 & 0.95 \\
\hline 13 & 1 & 1 & 1 & 0.86 & 0.85 \\
\hline 14 & 1 & 1 & 1 & 0.61 & 0.68 \\
\hline 15 & 3 & 3 & 3 & 0.82 & 0.85 \\
\hline 16 & 4 & 5 & 4 & 0.93 & 1.00 \\
\hline 17 & 3 & 2 & 3 & 0.93 & 0.83 \\
\hline 18 & 4 & 3 & 4 & 0.86 & 0.85 \\
\hline 19 & 1 & 1 & 1 & 0.89 & 0.80 \\
\hline 20 & 3 & 4 & 3 & 0.86 & 0.88 \\
\hline 21 & 1 & 1 & 1 & 0.75 & 0.78 \\
\hline 22 & 1 & 1 & 1 & 0.89 & 0.87 \\
\hline 23 & 1 & 1 & 1 & 0.86 & 0.85 \\
\hline 24 & 6 & 3 & 2 & 0.79 & 0.82 \\
\hline 25 & 4 & 3 & 3 & 0.86 & 0.88 \\
\hline 26 & 2 & 1 & 1 & 0.86 & 0.83 \\
\hline 27 & 5 & 4 & 4 & 0.93 & 0.82 \\
\hline 28 & 5 & 5 & 4 & 1.00 & 0.98 \\
\hline 29 & 4 & 3 & 1 & 0.93 & 0.92 \\
\hline 30 & 4 & 7 & 3 & 0.89 & 0.93 \\
\hline 31 & 6 & 6 & 5 & 0.89 & 0.80 \\
\hline 32 & 3 & 2 & 2 & 0.96 & 0.90 \\
\hline
\end{tabular}

Table 2 shows the three-years result of PROG and the score of "Daifuku-cho" of each student when they were in the 2nd grade and in the 4th grade. The score of "Daifuku-cho" is the percentage. The students from 1 to 12 show the improvement of their competency in two years from their 2nd grade to 4th grade (Up group). The students from 13 to 23 do not show any change in their competency (Even group). The students from 24 to 32 show drop in their competency scores (Down group). 
IADIS International Journal on Computer Science and Information Systems

Out of the 12 students in the Up group, eight students got better in "Daifuku-cho" assessment, but four did not change. Five students out of 11 Even group members got better, but the other six dropped in "Daifuku-cho." Three out of nine of the Down group got better, but the other six dropped. Figure 3 shows the percentages in each group. The percentage of students who got better is the highest, which is $67 \%$, in (a). $45 \%$ of them got better in (b), and $33 \%$ of them in (c), which is the lowest among three groups. We can see the improvement of "Daifuku-cho" grades in the order of (a), (b) and (c).

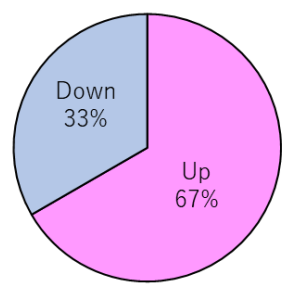

(a) Up

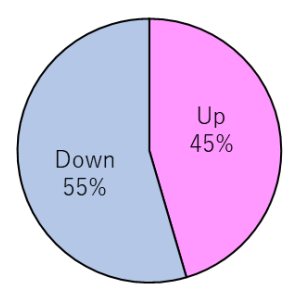

(b) Even

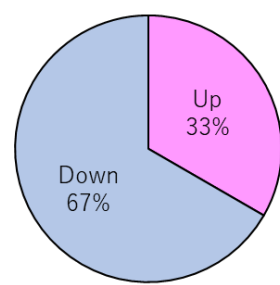

(c) Down

Figure 3. Relationship between PROG competency and grade in Daifuku-cho

Figure 4 shows the amount of change in students' grades of each group between their 2nd year and 4th year in box-and-whisker plot. The boxes show interquartile range (Q25-Q75) of each group, the bold lines the Median, the upper whiskers Max, the lower whiskers Min, and the dots indicate outliers. As it shows, the Up group got better in the average grade. Therefore, those who improved in their competency showed the tendency to get better in "Daifuku-cho" scores, while those who decreased in their competency scores had the tendency to go down in the average scores. The result of Tukey-Kramer test shows that there are significant differences in the average scores between Up group and Down group using a level of significance of $\mathrm{p}<0.2$.

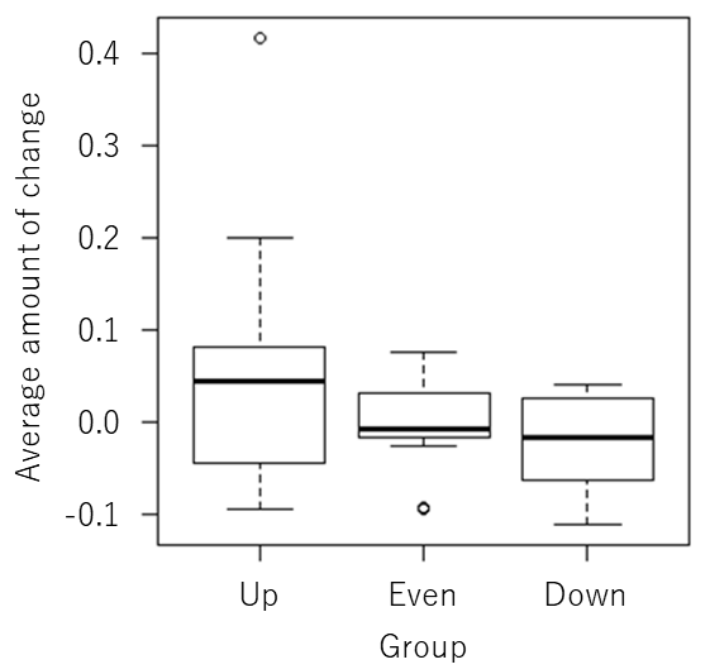

Figure 4. Competency and improvement in grade 


\subsection{Discussion}

The results of the present study indicate that the better the students get in their competency, the better their "Daifuku-cho" grades become, and vice versa.

We focused on the relationship of "Daifuku-cho" scores and "Competency" in PROG, and assessed students' grades in PE class. Assessing students' athletic skills is a regular part of PE, but on the other hand, it is relatively difficult to assess students' cognitive skills in PE class. We, however, were able to show the possibility of assessing the improvement in students' competency through the use of "Daifuku-cho."

Ito et al. shows that there is a weak correlation between GPA and PROG [Ito 2014]. In the present study, we also found out that there was a weak correlation between PROG and PE class grades, which suggests that we can assess the improvement in students' competency through the assessment by "Daifuku-cho."

So far, "Daifuku-cho" has been used as a communication tool between teachers and students, and also as a reflective tool for students. Our present study shows another potential of "Daifukucho" as a tool to measure the improvement in students' competency.

The present study deals with PE class, in which students' cognitive capacity does not lead to their grades straightforward, and considers the effectiveness of using "Daifuku-cho," which helps students to reflect themselves after each class and which students and their teacher can use to communicate with each other. There are various tools and methods to support students in learning proactively by encouraging reflective thinking, for example, the minute paper, questionnaire, or reflective journal, as well as "Daifuku-cho." Each tool has advantages and disadvantages. It is more important to choose right tools or methods to use at a right time and to construct effective lessons and curriculum depending on what ability of students we want to improve.

\section{CONCLUSION}

"Daifuku-cho" is used as a tool for students' own review of daily learning and teachers' feedback. The present study aims to investigate the learning effects by "Daifuku-cho" in terms of individual student's growth in competency.

We conducted a two-year follow-up review of 32 students and examined the correlation of grading by "Daifuku-cho" in PE class and the quantitative evaluation of their competency using PROG test. The students were divided into three groups: (a) those whose competency scores improved in two years, (b) those whose competency scores showed no change, and (c) those whose competency scores dropped. In "Daifuku-cho" assessment the percentage of students who got better is the highest, which is $67 \%$, in (a). $45 \%$ of them got better in (b), and $33 \%$ of them in (c), which is the lowest among three groups. We can see the improvement of "Daifuku-cho" grades in the order of (a), (b) and (c). And there were significant differences among the average scores of "Daifuku-cho" of each group and those whose competency scores improved also showed improvement in their "Daifuku-cho" scores.

These suggest a possibility that we could estimate a student's competency improvement according to the evaluation of his/her "Daifuku-cho." 
IADIS International Journal on Computer Science and Information Systems

\section{REFERENCES}

Confederation of British Industry, 1998. "Qualified to compete Creating a world class qualifications framework," Human Resources Brief, January, London: CBI.

Conference Board of "Employability Skills," https://www.conferenceboard.ca/edu/employability-skills.aspx

Fernandes, S.R.G., 2014. 'Preparing graduates for professional practice: findings from a case study of Project-based Learning (PBL),' Procedia - Social and Behavioral Sciences, Vol. 139, pp. 219-226.

Fujiki M.N. et al, 2016. "The practical program for bringing out generic skills of the first year students," TALE, pp.271-277, Dec., 2016.

Hassan, N.F. et al, 2015. 'Student understanding through the application of technology enabled Active Learning in practical training,' Procedia - Social and Behavioral Sciences, Vol. 204, pp. 318-325.

Ito H., 2014. "Assessing an Assessment Tool of Higher Education: Progress Report on Generic Skills (PROG) in Japan," IJERE, Vol.3, No.1, pp.1 -10.

Kawaijuku and Riasec, "PROG: Progress report on generic skills," https://www.kawaijuku.jp/jp/research/prog/point.html

Kogo C., 2006. "What Aspect of Class Did Shuttle Card "Daifukucho" Change?," JSET06-5, pp.23 - 30.

Kogo C., 2007. "Using Shuttle Card "Daifuku-cho” in e-Learning Courses," JSET07-05, pp. 297-300.

Kubota, Y. et al, 2017. "Analysis of Active Learning Suitability of Subjects in Information and Electronics," International Journal: Engineering Pedagogy, Vol.7, No.3, pp. 19-33.

Kularbphettonga K. et al, 2015. 'Developing an adaptive Web-Based Intelligent Tutoring System using Mastery Learning technique,' Procedia - Social and Behavioral Sciences, Vol. 191, pp. 686-691.

Kulik, C.L.C. et al, 1990. 'Effectiveness of Mastery Learning Programs: A Meta-Analysis,' REVIEW OF EDUCA'TIONAL RESEARCH Summer, Vol. 60 no.2 265-299.

Leung K. et al, 2014, "A study of the alignment of learning targets and assessment to generic skills in the new senior secondary mathematics curriculum in Hong Kong," Studies in Educational Evaluation, No. 43, pp.115-132.

MEXT, 2014, '2014 White Paper on Education, Culture, Sports, Science and Technology,' Ministry of Education, Culture, Sports, Science and Technology-JAPAN, http://www.mext.go.jp/b_menu/hakusho/html/hpab201401/1376911.htm

Niemi, H., 2002. 'Active learning — a cultural change needed in teacher education and schools,' Teaching and Teacher Education, vol. 18, pp. 763-780.

OECD, 2003. "Learning for Tomorrow's World, First Results from PISA." OECD.

OECD, 2005. "The Definition and Selection of Key Competencies (DeSeCo)," OECD.

Oda K., 1991. "Teaching Practice on the Effects of Shuttle-Card "Daifuku", Bulletin of Faculty of Education, Mie University Educational Science Educational Science (42), p165-174. (in Japanese)

Rodri'guez, J. et al, 2015. 'Project Based Learning experiences in the space engineering education at Technical University of Madrid,' Advances in Space Research, Vol. 56, pp. 1319-1330.

Shafie, N., et al, 2010. 'Mastery Learning Assessment Model (MLAM) in teaching and learning mathematics,' Procedia Social and Behavioral Sciences, Vol. 8, pp. 294-298.

Takahashi, A. et al, 2016. "A3 Learning System: Advanced Active and Autonomous Learning System," International Journal of Engineering Pedagogy, Vol.6, Issue2, pp. 52-58. 